\title{
Higher Order Sylster's Equation on Measure Chains-Controllability and Observability
}

\author{
Goteti V. R. L. Sarma \\ Department of Mathematics, University of Dodoma, Dodoma, Tanzania
}

Email address:

gvrlsarma@rediffmail.com

To cite this article:

Goteti V. R. L. Sarma. Higher Order Sylster's Equation on Measure Chains-Controllability and Observability. American Journal of Applied Mathematics. Vol. 3, No. 4, 2015, pp. 179-184. doi: 10.11648/j.ajam.20150304.13

\begin{abstract}
In this article we derive the solution of higher order Sylster's type differential equation on measure chains in terms of two fundamental matrices. Later by defining the controllability and observability on measure chains, necessary conditions for the controllability and observability of the higher order Sylster's type differential system on measure chains is established.
\end{abstract}

Keywords: Measure Chains, Controllability Observability, Fundamental Matrix

\section{Introduction}

Control theory has emerged into the main core of applied mathematical studies as it sets the necessary and sufficient conditions for controllability and observability of the designed dynamical systems. Many of the present day real world problems arises in robotics, industrial engineering, automata theory, modeling biological systems and in space dynamics are mostly control theoretic in nature as the aim being to compel or control the system to behave in some desired fashion. These systems can be continuous or discrete nature and the researchers earlier used to study them separately.

At the progress and advancement of knowledge on time scales (or measure chains) which includes both continuous and discrete systems as special cases make it possible to study the complex dynamical systems rigorously. Stephan Barnett [1] studied the control theory for both the continuous and discrete cases for simple dynamical systems. Many problems of great importance in the contemporary world require a quite different approach, the aim being to compel or control a system to behave in some desired fashion. Basically control theory has involved the study of analysis and control of any dynamical system. This theory has been successfully applied in a variety of branches in the disciplines of engineering and particularly it is receiving great impetus from Aerospace engineering. A fascinating fact is that all the widely different disciplines of applications depend on a common core of mathematical techniques of the modern control system theory. The results established in this article coincide with the findings of $[1],[2],[5]$ and [11] and include them as a sub case of this article.

In this paper we establish the concept of controllability and observability of dynamical systems on measure chains. The results presented in this chapter generalises the existing results on controllability and observability for continuous and discrete cases and includes them as a particular case. This paper is organised as follows: In section 2, we outline the salient features of time scales. Section 3, deals with the method of solution of the initial value problem on higher order Sylster's type equation on measure chains

$$
X^{\Delta^{n}}(t)=\sum_{r=0}^{n} n_{c_{r}} A^{n-r} X(\sigma(t)) B^{r}+F(t, X(t)), X\left(t_{0}\right)=X_{0}
$$

In terms of two fundamental matrix solutions, finally in section 4 , we obtain the necessary and sufficient conditions for the controllability and observability of higher order Sylster's type matrix dynamical systems on measure chains

$$
S\left\{\begin{array}{c}
X^{\Delta^{n}}(t)=\sum_{r=0}^{n} n_{c_{r}} A^{n-r} X(\sigma(t)) B^{r}+C(t) U(t), X\left(t_{0}\right)=X_{0} \\
Q(t)=P(t) X(t)
\end{array}\right.
$$

Where $\mathrm{A}$ and $\mathrm{B}$ are constant square matrices of order $n$ and $X, C, U, P \in\left[R^{+}, R^{n} X R^{n}\right]$ are all variable matrics whose elements are rd-continuous on a measure chain $\mathrm{T}=\left[\mathrm{t}_{0}, \mathrm{t}_{\mathrm{N}}\right]$. We firmly believe that these results will have a significant impact on robotic and control engineering applications. 


\section{Salient Features of Time Scales}

A measure chain (or time scale) is an arbitrary closed subset of real numbers $\mathrm{R}$ and it is denoted by $\mathrm{T}$ throughout the paper. Time scales are not necessarily connected and this topological handicap is eliminated by introducing the notion of jump operators $\sigma$ and $\rho$ as follows:

Definition 2.1: Let $\mathrm{T}$ be a measure chain. For $\mathrm{t} \in \mathrm{T}$ define the forward jump operator $\sigma: T \rightarrow T$ by $\sigma(t)=\operatorname{Inf}\{s \in T: s>$ $\mathrm{t}\}$ and the backward jump operator $\rho: \mathrm{T} \rightarrow \mathrm{T}$ by $\rho(\mathrm{t})=\operatorname{Sup}\{\mathrm{s}$ $\in \mathrm{T}: \mathrm{s}<\mathrm{t}\}$.

A point $t \in T$ is said to be right dense, right scattered, left dense and left scattered according as $\sigma(\mathrm{t})=\mathrm{t}, \sigma(\mathrm{t})>\mathrm{t}, \rho(\mathrm{t})=\mathrm{t}$ and $\rho(\mathrm{t})<\mathrm{t}$ respectively.

The grainness $\mu: T \rightarrow[0, \infty)$ is defined by $\mu(t)=\sigma(t)-t$.

The set $T^{k}$ which is derived from the measure chain $T$ as follows:

If $\mathrm{T}$ has a left scattered maximum $\mathrm{m}$, then $\mathrm{T}^{\mathrm{k}}=\mathrm{T}-\{\mathrm{m}\}$, otherwise $\mathrm{T}^{\mathrm{k}}=\mathrm{T}$.

Definition 2.2: Let $\mathrm{f}: \mathrm{T} \rightarrow \mathrm{R}, \mathrm{t} \in \mathrm{T}^{\mathrm{k}}$. Then define $\mathrm{f}^{\mathrm{A}}(\mathrm{t})$ to be the number (provided it exists ) with the property that given any $\in>0$, there exists a neighbourhood $\cup$ of $t$ such that

$$
\left|[\mathrm{f}(\sigma(\mathrm{t}))-\mathrm{f}(\mathrm{s})]-\mathrm{f}^{\Delta}(\mathrm{t})[\sigma(\mathrm{t})-\mathrm{s}]\right| \leq \in|\sigma(\mathrm{t})-\mathrm{s}| \text { for all } \mathrm{s} \in \cup
$$

Then $\mathrm{f}^{\Delta}(\mathrm{t})$ is called the delta derivative of $\mathrm{f}$ at $\mathrm{t}$.

If $\mathrm{T}=\mathrm{R}$, the delta derivative is same as that of ordinary derivative and for $\mathrm{T}=\mathrm{Z}, \mathrm{f}^{\Delta}(\mathrm{t})=\mathrm{f}(\mathrm{t}+1)-\mathrm{f}(\mathrm{t})=\Delta \mathrm{f}(\mathrm{t})$, which is the forward difference operator.

Definition 2.3: We say that $\mathrm{f}$ is delta differentiable on $\mathrm{T}^{\mathrm{k}}$, if $\mathrm{f}^{\Delta}(\mathrm{t})$ exists for all $\mathrm{t} \in \mathrm{T}^{\mathrm{k}}$.

Result 2.1: Assume $\mathrm{f}, \mathrm{g}: \mathrm{T} \rightarrow \mathrm{R}$ are delta differentiable functions at $\mathrm{t} \in \mathrm{T}^{\mathrm{k}}$, then

(i) $\mathrm{f}+\mathrm{g}: \mathrm{T} \rightarrow \mathrm{R}$ is delta differentiable at $\mathrm{t}$ with $(\mathrm{f}+\mathrm{g})^{\Delta}(\mathrm{t})=$ $\mathrm{f}^{\Delta}(\mathrm{t})+\mathrm{g}^{\Delta}(\mathrm{t})$.

(ii) For any constant $\mathrm{k}, \mathrm{kf}: \mathrm{T} \rightarrow \mathrm{R}$ is delta differentiable at $\mathrm{t}$ with $(\mathrm{kf})^{\Delta}(\mathrm{t})=\mathrm{k} \mathrm{f}^{\Delta}(\mathrm{t})$.

(iii) $\mathrm{fg}: \mathrm{T} \times \mathrm{T} \rightarrow \mathrm{R}$ is delta differentiable at $\mathrm{t}$ with

$$
\begin{gathered}
(\mathrm{fg})^{\Delta}(\mathrm{t})=\mathrm{f}^{\Delta}(\mathrm{t}) \mathrm{g}(\mathrm{t})+\mathrm{f}(\sigma(\mathrm{t})) \mathrm{g}^{\Delta}(\mathrm{t})=\mathrm{f}(\mathrm{t}) \mathrm{g}^{\Delta}(\mathrm{t})+ \\
\mathrm{f}^{\Delta}(\mathrm{t}) \mathrm{g}(\sigma(\mathrm{t})) .
\end{gathered}
$$

Result 2.2: Leibnitz like theorem on measure chains: If $\mathrm{f}(\mathrm{t})$ and $\mathrm{g}(\mathrm{t})$ are continuously $n$ times delta differentiable functions on a measure chain $\mathrm{T}=[\mathrm{a}, \mathrm{b}]$ then

$$
(f \cdot g)^{\Delta^{n}}(t)=\sum_{r=0}^{n} n C_{r} f^{\Delta^{n-r}}\left(\sigma^{r}(t)\right) g^{\Delta^{r}}(t)
$$

Definition 2.4: A function $\mathrm{F}: \mathrm{T}^{\mathrm{k}} \rightarrow \mathrm{R}$ is called an antiderivative of $\mathrm{f}: \mathrm{T}^{\mathrm{k}} \rightarrow \mathrm{R}$, provided $\mathrm{F}^{\Delta}(\mathrm{t})=\mathrm{f}(\mathrm{t})$ holds for all $\mathrm{t}$ $\in \mathrm{T}^{\mathrm{k}}$. Then the delta integral of $\mathrm{f}$ is defined $=\mathrm{F}(\mathrm{t})-\mathrm{F}(\mathrm{a}) \forall \mathrm{t} \in$ $\mathrm{T}$.

Definition 2.5: Let $\mathrm{f}: \mathrm{T} \rightarrow \mathrm{T}$ be a function. We say that $\mathrm{f}$ is $\mathrm{rd}$ - continuous if it is continuous in right dense points and if limit $f(s)$ exists as $s \rightarrow t^{-}$for all left-dense points $t \in T$.

Result $2.3: \mathrm{Rd}$ - Continuous functions possess an anti derivative.

Proof: For the proof we refer [2].

\section{Solving Higher order Syslster's Equation}

Throughout this article $Y(t)$ denotes the fundamental matrix solution of $X^{\Delta}(t)=A X(t)$ and $\mathrm{Z}(\mathrm{t})$ denotes the fundamental matrix solution of $X^{\Delta}(t)=B^{*} X(\sigma(t))$.

Theorem 3.1: If $Y(t)$ is a fundamental matrix solution of $X^{\Delta}(t)=A X(t)$ then $Y(t)$ is also fundamental matrix solution of $X^{\Delta^{n}}(t)=A^{n} X(t)$.

Proof: $Y(t)$ is a fundamental matrix solution of $X^{\Delta}(t)=$ $A X(t)$

This implies $Y^{\Delta}(t)=A Y(t)$

Delta differentiating on both sides gives

$$
Y^{\Delta^{2}}(t)=A Y^{\Delta}(t) \Rightarrow Y^{\Delta^{2}}(t)=A^{2} Y(t)
$$

Similarly $Y^{\Delta^{3}}(t)=A^{3} Y(t)$

Continuing like this we can conclude that $Y^{\Delta^{n}}(t)=$ $A^{n} Y(t)$

Hence $Y(t)$ is also fundamental matrix solution of $X^{\Delta^{n}}(t)=A^{n} X(t)$.

Theorem 3.2: Any solution of

$$
X^{\Delta^{n}}(t)=\sum_{r=0}^{n} n_{c_{r}} A^{n-r} X(\sigma(t)) B^{r}
$$

is of the form $X(t)=Y(t) C Z^{*}(t)$ where $\mathrm{C}$ is any square matrix of order $n$.

Proof: Using Lebnitz like theorem on measure chains for the $n^{\text {th }}$ delta derivative of product of functions we have

$$
\begin{aligned}
& \left(Y(t) C Z^{*}(t)\right)^{\Delta^{n}}=Y^{\Delta^{n}}(t) C Z^{*}(t)+n_{c_{1}} Y^{\Delta^{n-1}}(\sigma(t)) C Z^{*^{\Delta}}(t)+n_{c_{2}} Y^{\Delta^{n-2}}(\sigma(t)) C Z^{*^{\Delta^{2}}}(t)+\ldots+Y(\sigma(t)) C Z^{*^{\Delta^{n}}}(t) \\
& =A^{n} Y(t) C Z^{*}(t)+n_{c_{1}} A^{n-1} Y(\sigma(t)) C Z^{*}(\sigma(t)) B+n_{c_{2}} A^{n-2} Y(\sigma(t)) C Z^{*}(\sigma(t)) B^{2}+\ldots+Y(\sigma(t)) C Z^{*}(\sigma(t)) B^{n} \\
& =A^{n} X(t)+n_{c_{1}} A^{n-1} X(\sigma(t)) B+n_{c_{2}} A^{n-2} X(\sigma(t)) B^{2}+\cdots+X(\sigma(t)) B^{n}=\sum_{r=0}^{n} n_{c_{r}} A^{n-r} X(\sigma(t)) B^{r}
\end{aligned}
$$

Hence $X(t)=Y(t) C Z^{*}(t)$ is a solution of $(3.1)$

To prove that every solution of (3.1) is of this form, let $\mathrm{X}(\mathrm{t})$ be a solution of defined by $X(t)=Y(t) K(t)$ where $K(t)$ is any variable matrix of order $n$.

Then using Leibnitz like theorem we have

$$
(Y(t) K(t))^{\Delta^{n}}=Y^{\Delta^{n}}(t) K(t)+n_{c_{1}} Y^{\Delta^{n-1}}(\sigma(t)) K^{\Delta}(t)+\cdots+n_{c_{r}} Y^{\Delta^{n-r}}(\sigma(t)) K^{\Delta^{r}}(t)+\ldots+Y(\sigma(t)) K^{\Delta^{n}}(t)
$$




$$
=A^{n} Y(t) K(t)+n_{c_{1}} A^{n-1} Y(\sigma(t)) K^{\Delta}(t)+\cdots+Y(\sigma(t)) K^{\Delta^{n}}(t)
$$

This is equivalent to R.H.S. of (3.1) if $K^{\Delta}(t)=K(\sigma(t)) B$

$$
\text { i.e. } K^{*}(t)=B^{*} K^{*}(\sigma(t))
$$

i.e. $K^{*}$ is a solution of $X^{\Delta}(t)=B^{*} X(\sigma(t))$

Since $Z(\mathrm{t})$ is the fundamental matrix solution of $X^{\Delta}(t)=$ $B^{*} X(\sigma(t))$ hence $K^{*}(t)=Z(t) C^{*}(t)$

$$
\text { Hence } K(t)=C(t) Z^{*}(t)
$$

Hence $(t)=Y(t) C Z^{*}(t)$. This completes the proof. Theorem 3.3: Any solution of the initial value problem

$$
X^{\Delta^{n}}(t)=\sum_{r=0}^{n} n_{c_{r}} A^{n-r} X(\sigma(t)), X\left(t_{0}\right)=X_{0}
$$

is given by

$$
X(T)=Y\left[t, t_{0}\right] X_{0} Z^{*}\left[t_{0}, t\right]
$$

where $Y\left[t, t_{0}\right]=Y(t) Y^{-1}\left(t_{0}\right)$ and $Z\left[t, t_{0}\right]=Z(t) Z^{-1}\left(t_{0}\right)$ Proof: From theorem 3.2 any solution of (3.1) is of the form $X(t)=Y(t) C Z^{*}(t)$ where $\mathrm{C}$ is any square matrix of order $n$.

Now substituting $X\left(t_{0}\right)=X_{0}$ gives $X_{0}=Y\left(t_{0}\right) C Z^{*}\left(t_{0}\right)$

Hence $C=Y^{-1}\left(t_{0}\right) X_{0} Z^{*^{-1}}\left(t_{0}\right)$

Hence the solution of the above initial value problem is $X(T)=Y\left[t, t_{0}\right] X_{0} Z^{*}\left[t_{0}, t\right]$ where $Y\left[t, t_{0}\right]=Y(t) Y^{-1}\left(t_{0}\right)$ and $Z\left[t, t_{0}\right]=Z(t) Z^{-1}\left(t_{0}\right)$. Hence the proof.

Theorem 3.4: Let $Y(t)$ be a fundamental matrix solution of $X^{\Delta}(t)=A X(t)$ and $\mathrm{Z}(\mathrm{t})$ be a fundamental matrix solution of $X^{\Delta}(t)=B^{*} X(\sigma(t))$. Further suppose that the variable matrix $C(t)$ is such that

$$
\left(Y ^ { \Delta } \left(\sigma^{i-1}(t) C^{\Delta}(t) Z^{*}(t)+Y\left(\sigma^{i}(t) C^{\Delta}\left(\sigma^{i-1}(t)\right) Z Z^{* \Delta}(t)\right)^{\Delta^{i-2}}=0\right.\right.
$$

for each $i=2,3, \ldots n$.

Then the particular solution of

$$
X^{\Delta^{n}}(t)=\sum_{r=0}^{n} n_{c_{r}} A^{n-r} X(\sigma(t)) B^{r}+F(t, X(t))
$$

is

$$
\bar{X}=Y(t)\left[\int_{a}^{t} \int_{a}^{\tau_{1}} \ldots \int_{a}^{\tau_{n-1}} Y^{-1}\left(\sigma^{*}(s) F(s, X(s)) Z^{*^{-1}}(s) \Delta s \Delta \tau_{n-1} \ldots \Delta \tau_{1}\right] Z^{*}(t)\right.
$$

Proof: Any solution of the corresponding homogeneous equation of (3.3) is in the form $X(t)=Y(t) C Z^{*}(t)$. Where $\mathrm{C}$ is any constant square matrix of order $n$. Since such a solution can not be the solution of non homogeneous equation (3.3), by variation of parameters formula we can assume the $\mathrm{C}$ is a variable matrix and seek the particular solution of $(3.3)$ in the form $X(t)=$ $Y(t) C(t) Z^{*}(t)$

Substituting this in the equation (3.3) and using the condition (3.2) we get

$$
Y(t) C^{\Delta^{(n)}}(t) Z^{*}(t)=F(t, X(t)) \Rightarrow C^{\Delta^{(n)}}(t)=Y^{-1}(t) F(t, X(t)) Z^{*^{-1}}(t)
$$

By delta integrating $n$ times on both sides we get

$$
C(t)=\left[\int_{a}^{t} \int_{a}^{\tau_{1}} \ldots \int_{a}^{\tau_{n-1}} Y^{-1}\left(\sigma^{*}(s) F(s, X(s)) Z^{*^{-1}}(s) \Delta s \Delta \tau_{n-1} \ldots \Delta \tau_{1}\right]\right.
$$

Hence the particular solution of (3.2) is

$$
\bar{X}=Y(t)\left[\int_{a}^{t} \int_{a}^{\tau_{1}} \ldots \int_{a}^{\tau_{n-1}} Y^{-1}\left(\sigma^{*}(s) F(s, X(s)) Z^{*^{-1}}(s) \Delta s \Delta \tau_{n-1} \ldots \Delta \tau_{1}\right] Z^{*}(t)\right.
$$

Theorem 3.5: Any solution of the non homogeneous system (3.2) is of the form

$$
\mathrm{X}(\mathrm{t})=Y(t) C Z^{*}(t)+Y(t)\left[\int_{a}^{t} \int_{a}^{\tau_{1}} \ldots \int_{a}^{\tau_{n-1}} Y^{-1}\left(\sigma^{*}(s) F(s, X(s)) Z^{*^{-1}}(s) \Delta s \Delta \tau_{n-1} \ldots \Delta \tau_{1}\right] Z^{*}(t)\right.
$$

Proof: Follows from the theorems 3.2 and 3.4

Theorem 3.6: Any solution of the initial value problem

$$
X^{\Delta^{n}}(t)=\sum_{r=0}^{n} n_{c_{r}} A^{n-r} X(\sigma(t)) B^{r}+F(t, X(t)), X\left(t_{0}\right)=X_{0}
$$

is given by 


$$
X(T)=Y\left[t, t_{0}\right] X_{0} Z^{*}\left[t_{0}, t\right]+Y\left[t, t_{0}\right]\left[\int_{t_{0}}^{t} \int_{t_{0}}^{\tau_{1}} \ldots \int_{t_{0}}^{\tau_{n-1}} Y\left[t_{0}, \sigma^{*}(s)\right] F(s, X(s)) Z^{*}\left[s, t_{0}\right] \Delta s \Delta \tau_{n-1} \ldots \Delta \tau_{1}\right] Z^{*}\left[t_{0}, t\right]
$$

Proof : Follows from the theorem 3.3 and 3.4

\section{Controllability and Observability}

In this section we consider the higher order Sylster's type matrix dynamical systems on measure chains

$$
X^{\Delta^{n}}(t)=\sum_{r=0}^{n} n_{c_{r}} A^{n-r} X(\sigma(t)) B^{r}+C(t) U(t), X\left(t_{0}\right)=X_{0}
$$

and obtain the necessary and sufficient conditions for the controllability and observability of the corresponding control engineering system

$$
\begin{gathered}
S\left\{\begin{array}{c}
X^{\Delta^{n}}(t)=\sum_{r=0}^{n} n_{c_{r}} A^{n-r} X(\sigma(t)) B^{r}+C(t) U(t), X\left(t_{0}\right)=X_{0} \\
Q(t)=P(t) X(t) \\
X(T)=Y\left[t, t_{0}\right] X_{0} Z^{*}\left[t_{0}, t\right]+Y\left[t, t_{0}\right]\left[\int_{t_{0}}^{t} \int_{t_{0}}^{\tau_{1}} \cdots \int_{t_{0}}^{\tau_{n-1}} Y\left[t_{0}, \sigma^{*}(s)\right] C(s) U(s) Z^{*}\left[s, t_{0}\right] \Delta s \Delta \tau_{n-1} \ldots \Delta \tau_{1}\right] Z^{*}\left[t_{0}, t\right] \\
=Y\left[t, t_{0}\right]\left[X_{0}+\left[\int_{t_{0}}^{t} \int_{t_{0}}^{\tau_{1}} \ldots \int_{t_{0}}^{\tau_{n-1}} Y\left[t_{0}, \sigma^{*}(s)\right] C(s) U(s) Z^{*}\left[s, t_{0}\right] \Delta s \Delta \tau_{n-1} \ldots \Delta \tau_{1}\right]\right] Z^{*}\left[t_{0}, t\right]
\end{array}\right.
\end{gathered}
$$

Definition 4.1 : The higher order Sylster's type time varying dynamical system $\mathrm{S}$ on measure chain $\mathrm{T}=\left[\mathrm{t}_{0}, \mathrm{t}_{\mathrm{N}}\right]$ is said to be completely controllable if "for any initial time $\mathrm{t}_{0}$ and any initial state $x\left(t_{o}\right)=x_{o}$ and any given final state $x_{f}$, there exists a finite time $t_{N}>t_{o}$ and a control $u(t), t_{0} \leq t \leq t_{N}$ such that $x\left(t_{N}\right)=$ $\mathrm{x}_{\mathrm{f}}$ ".

Definition 4.2: The higher order Sylster's type time varying dynamical system defined by $\mathrm{S}$ on measure chains is said to be completely observable if and only if the knowledge of the control $u(t)$ and the out put $Q(t)$ suffice to determine $x\left(t_{0}\right)=x_{0}$ uniquely for a finite time $t_{N} \geq t_{0}$.

Proof: Follows from theorem 3.6.

Theorem 4.2: The Lyapunov type matrix dynamical system on measure chains $S$ is completely controllable if and only if the symmetric controllability matrix

$$
W\left[t_{0}, t_{N}\right]=\left[\int_{t_{0}}^{t_{N}} \int_{t_{0}}^{\tau_{1}} \ldots \int_{t_{0}}^{\tau_{n-1}} Y\left[t_{0}, \sigma^{*}(s)\right] C(s) C^{*}(s) Y^{*}\left[\sigma(s), t_{0}\right] \Delta s \Delta \tau_{n-1} \ldots \Delta \tau_{1}\right]
$$

is non singular.

Then the control $\mathrm{U}(\mathrm{t})$ defined by

$$
U(t)=-C^{*}(t) Y^{*}\left[t_{0}, \sigma(t)\right] W^{-1}\left[t_{0}, t_{N}\right]\left[X_{0}-Y\left[t_{0}, t_{N}\right] X_{F} Z^{*}\left[t_{0}, t_{N}\right]\right] Z^{*^{-1}}\left[t_{0}, t\right]
$$

defined for $\mathrm{t}_{0}<\mathrm{t}<\mathrm{t}_{\mathrm{N}}$ transfers $\mathrm{X}\left(\mathrm{t}_{0}\right)=\mathrm{X}_{0}$ to $\mathrm{X}\left(\mathrm{t}_{\mathrm{N}}\right)=\mathrm{X}_{\mathrm{f}}$.

Proof: First we suppose that $W\left[t_{0}, t_{N}\right]$ is non - singular. Then $U(t)$ defined as above exists. We know that any solution of (4.1) has the form

$$
X(t)=Y\left[t, t_{0}\right]\left[X_{0}+\left[\int_{t_{0}}^{t} \int_{t_{0}}^{\tau_{1}} \ldots \int_{t_{0}}^{\tau_{n-1}} Y\left[t_{0}, \sigma^{*}(s)\right] C(s) U(s) Z^{*}\left[s, t_{0}\right] \Delta s \Delta \tau_{n-1} \ldots \Delta \tau_{1}\right]\right] Z^{*}\left[t_{0}, t\right]
$$

put $t=t_{N}$ and substitute $U(t)$ defined as above we will get $X\left(t_{N}\right)=X_{f}$ and hence $S$ is controllable.

Conversely, suppose that $S$ is controllable. We have to show that $W\left[t_{0}, t_{N}\right]$ is non-singular.

Since $W\left[t_{0}, t_{N}\right]$ is symmetric, clearly it is positive semi definite.

Now suppose that there exists some column vector $\Psi \neq 0$ such that

$$
\Psi^{*} \mathrm{~W}\left[\mathrm{t}_{0}, \mathrm{t}_{\mathrm{N}}\right] \Psi=0 \Rightarrow \int_{t_{0}}^{t_{N}} \Psi^{*} \mathrm{~W}\left[\mathrm{t}_{0}, \sigma(\mathrm{s})\right] \mathrm{C}(\mathrm{s}) \mathrm{C}^{*}(\mathrm{~s}) \mathrm{W}^{*}\left[\mathrm{t}_{0}, \sigma(\mathrm{s})\right] \Psi \Delta s=0 \Rightarrow \int_{t_{0}}^{t_{N}} \theta^{*}\left[\mathrm{~s}, \mathrm{t}_{0}\right] \theta\left[s, t_{0}\right] \Delta s=0
$$

where

$$
\theta\left[s, t_{0}\right]=\mathrm{C}^{*}(\mathrm{~s}) \mathrm{W}^{*}\left[\mathrm{t}_{0}, \sigma(\mathrm{s})\right] \Psi \Rightarrow \int_{t_{0}}^{t_{N}}\left\|\theta^{2}\right\|^{*} \Delta s=0 \text { Hence } \theta=0 \text { on }\left[t_{0}, t_{N}\right] .
$$

By our assumption that $S$ is completely controllable, there exists a control $\mathrm{V}(\mathrm{t})$ (say) making $X\left(t_{N}\right)=0$ if $X\left(t_{0}\right)=\Psi g$ 
where $g$ is any non zero $1 \mathrm{Xn}$ matrix.

$$
\begin{aligned}
X\left(t_{N}\right) & =0 \Rightarrow X_{0}+\left[\int_{t_{0}}^{t_{N} t} \int_{t_{0}}^{\tau_{1}} \ldots \int_{t_{0}}^{\tau_{n-1}} Y\left[t_{0}, \sigma^{*}(s)\right] C(s) U(s) Z^{*}\left[s, t_{0}\right] \Delta s \Delta \tau_{n-1} \ldots \Delta \tau_{1}\right]=0 \\
& \Rightarrow \Psi g=-\left[\int_{t_{0}}^{t_{N}} \int_{t_{0}}^{\tau_{1}} \ldots \int_{t_{0}}^{\tau_{n-1}} Y\left[t_{0}, \sigma^{*}(s)\right] C(s) U(s) Z^{*}\left[s, t_{0}\right] \Delta s \Delta \tau_{n-1} \ldots \Delta \tau_{1}\right]
\end{aligned}
$$

Now it can be easily shown that $\|\Psi g\|=(\Psi g)^{*}(\Psi g)=0$ Therefore $\Psi g=0$ Hence $\Psi=0$ which is a contradiction.

Therefore $W\left[t_{0}, t_{N}\right]$ is a positive definite matrix hence it is non-singular.

Theorem 4.3: The system $S$ is completely observable if and only if the symmetric observability matrix

$$
V\left(t_{0}, t_{N}\right)=\int_{t_{0}}^{t_{N}} Y^{*}\left[\sigma(s), t_{0}\right] P^{*}(s) P(s) Y\left[\sigma(s), t_{0}\right] \Delta s
$$

is non singular.

Proof: Suppose $V\left(t_{0}, t_{N}\right)$ is nonsingular.

Without loss of generality suppose that $\mathrm{U}(\mathrm{t}) \equiv 0 \forall \mathrm{t} \in\left[\mathrm{t}_{0}, \mathrm{t}_{\mathrm{N}}\right]$ Then

$$
X(t)=Y\left[t, t_{0}\right] X_{0} Z^{*}\left[t, t_{0}\right]
$$

For this the output is

$$
\begin{gathered}
Q(t)=P(t) Y\left[t, t_{0}\right] X_{0} Z^{*}\left[t, t_{0}\right] \Rightarrow \\
Y^{*}\left[t, t_{0}\right] P^{*}(t) Q(t) Z^{*^{-1}}\left[t, t_{0}\right]=Y^{*}\left[t, t_{0}\right] P^{*}(t) P(t) Y\left[t, t_{0}\right] X_{0}
\end{gathered}
$$

Delta integrating from $t_{0}$ to $t_{N}$ we get

$$
\int_{t_{0}}^{t_{N}} Y^{*}\left[s, t_{0}\right] P^{*}(s) Q(s) Z^{*^{-1}}\left[s, t_{0}\right] \Delta s=V\left[t, t_{N}\right] X_{0}
$$

Which gives

$$
X_{0}=V^{-1}\left[t_{0}, t_{N}\right] \int_{t_{0}}^{t_{N}} Y^{*}\left[s, t_{0}\right] P^{*}(s) Q(s) Z^{*^{-1}}\left[s, t_{0}\right] \Delta s
$$

Therefore $S$ is completely observable.

Conversely suppose $S$ is completely observable.

We will show that $V\left(t_{0}, t_{N}\right)$ is non singular.

Since $V\left(t_{0}, t_{N}\right)$ is symmetric, clearly it is positive semi definite.

If possible suppose that there exists a column vector $\Phi \neq 0$ such that

$$
\begin{gathered}
\Phi^{*} V\left(t_{0}, t_{N}\right) \Phi=0 \text {. Then } \int_{t_{0}}^{t_{N}}\left\|P(s) Y\left[s, t_{0}\right] \Phi\right\|^{2} \Delta s=0 \\
\Rightarrow P(s) Y\left[s, t_{0}\right] \Phi=0 \forall s \in\left[\mathrm{t}_{0}, \mathrm{t}_{\mathrm{N}}\right] .
\end{gathered}
$$
is

If $X_{0}=\Phi K$ where $\mathrm{K}$ is a matrix of order $\mathrm{n}$, then the output

$$
Q(t)=P(t) Y\left[t, t_{0}\right] X_{0} Z^{*}\left[t, t_{0}\right]=P(t) Y\left[t, t_{0}\right] \Phi K Z^{*}\left[t, t_{0}\right]=0
$$

i.e $X_{0}$ can not determined with the knowledge of $Q(t)$ in this case. This contradicts our assumption that $S$ is completely observable.

Therefore $V\left(t_{0}, t_{N}\right)$ is positive definite and hence $V\left(t_{0}, t_{N}\right)$ is non - singular.

Observation 4.1: From the theorem (4.2) it is observed that the controllability matrix is independent of fundamental matrix $Z$. The fundamental matrix $Y$ alone determines the controllability criterion of the dynamical system $S$. We also observe that the controllability criterion can be determined using the fundamental matrix $Z$ alone through properly defined controllability matrix.

Observation 4.2: From the theorem (4.3) it is observed that the observability matrix is independent of $Z$. The fundamental matrix $Y$ alone determines the observability criterion of the dynamical system $S$.

\section{Summary and Conclusions}

These results coincide with the findings [1],[2],[5] and [11] of and include them as a sub case of this article. Hence this article generalizes the findings of the article and can be applicable to wider range of control engineering problems. Using these results we can study further class of differential systems on measure chains for their controllability and observability.

\section{References}

[1] Alaa E Hamza, Karima M Oraby, Stability of abstract dynamic equations on time scales, Advances in Difference Equations 2012, 2012:143

[2] Z. Bartosiewicz and E. Pawłuszewicz, Realizations of linear control systems on time scales, Control \& Cybernetics, vol. 35, no. 4, pp.769-786, 2006.

[3] J. Z. Bartosiewicz and E. Pawłuszewicz, Linear control systems on time scale: unification of continuous and discrete, in Proceedings of the 10th IEEE International Conference On Methods and Models in Automation and Robotics MMAR 2004, Miedzyzdroje, Poland, 2004.

[4] J.J. Da Cunha, Stability for time varying linear dynamic systems on time scales, J. Comput. Appl. Math. 176 (2005)381-410.

[5] V.Lakshmikantham, S.Sivasundaram and B.Kaymakclan, Dynamic systems on measure chains, Kluwer academic Publishers, (1996).

[6] R. J. Marks II, I. A. Gravagne, J. M. Davis, and J. J. Da Cunha, Nonregressivity in switched linear circuits and mechanical systems, Mathematical and Computer Modelling, vol.43, pp. 1383-1392, 2006.

[7] K.N.Murty, G.W.Howell and G.V.R.L. Sarma, Two (Multi) Point Nonlinear Lyapunov Systems Associated with an $\mathrm{n}^{\text {th }}$ order Nonlinear system of differential equations-Existence and Uniqueness, Journal of Mathematical Problems In Engineering, Vol 6, Pp 395-410, 2000. 
[8] K.N.Murty, Y.Narasimhulu and G.V.R.L.Sarma, Controllability and Observability of Lyapunov type Matrix Integro differential systems. International Journal of non linear differential equations: Theory, Methods and Applications, Vol 7, Nos 1\& 2, pp $1-18,2001$.

[9] Murty K.N., Rao Y.S Two point boundary value problems inhomogeneous time scale linear dynamic process, JMAA 184(1994) pp 22-34.

[10] Nguyen Huu Du and Le Huy Tien, On the exponential stability of dynamic equations on time scales, J. Math. Anal. Appl. 331 (2007) 1159-1174.
[11] Pitzsche, S. Sigmund, and F. Wirth, A spectral characterization of exponential stability for linear time-invariant systems on time scales, Discrete and Continuous Dynamical Systems, vol. 9, no. 5, pp. 1223-1241, 2003.

[12] Goteti V.R.L.Sarma, A new look into the Controllability and Observability of Lyapunov type matrix dynamical systems on measure chains, Global Journal of research in engineering (I) Vol XIV Issue II, Version 1, 2014, pp 5-8

[13] Stephen Barnet, Introduction to mathematical control, Clarendon Press- Oxford (1975). 